\title{
Research of UAV cooperative reconnaissance with self- organization path planning
}

\author{
Liu Minjia ${ }^{1,2}$, Lin Jie ${ }^{1,2}$, Yuan Yuyu',2 \\ ${ }^{1}$ School of Computer Science and Technology, Beijing University of Posts and Telecommunications, Beijing 100876, China \\ ${ }^{2}$ Key Laboratory of Trustworthy Distributed Computing and Services, Ministry of Education, Beijing University of Posts and \\ Telecommunications, Beijing 100876, China \\ liuminjiaofbupt@163.com
}

\begin{abstract}
Unmanned aerial vehicle (UAV) builds cognitive map for the enemy important targets and anti-aircraft fire threats in the unknown environment. Then UAV tries to avoid the threats and head to important targets where the enemy locate. This paper studies how the unmanned aerial vehicle (UAV) in complex dynamic field environment make path planning to search the enemy's important targets. The standard for choosing the most appropriate path from alternative paths is based on cost equation. It is the smallest cost that the UAV can get. The study of self organization path planning is to analyze the four kinds of short-term costs and a long-term cost. Through adjusting the weight coefficient, all kinds of UAV path plannings in different battlefield environment can be achieved.

Index Terms - unmanned aerial vehicles(UAV), cooperative reconnaissance, path planning, information distribution, selforganization

\section{Introduction}

Unmanned aerial vehicle (UAV) produced a profound influence on war. There is no human pilot on UAV, and UAV can perform real-time operation on the battlefield to avoid the loss of valuable human pilots' lives. In the high risk battlefield, the UAV can make long time reconnaissance against hostile forces. The UAV costs less when compared with the ordinary military aircraft, and there is no loss of human pilots.
\end{abstract}

\section{The self-organization path planning for UAV cooperative reconnaissance}

Cooperation of different types of UAV on the battlefield is needed, such as reconnaissance, fighter and bomber UAV, to complete more than one combat mission. UAV cognitive map divides the battlefield environment into different sub areas.

UAV searches around battlefield environment with a higher uncertainty. UAV scans battlefield environment in continuous to update cognitive map, in which the important enemies' military targets in this sub region are labeled as destination of the bomber UAV, and enemies' anti-aircraft fire defenses in this sub - region are marked as "soft obstacle". The ability to see and avoid obstacles is a necessary condition for UAV flight[1]. UAV bombers will attack the mission targets with path planning according to cognitive map while UAV bombers getting away from the "soft obstacles" to prevent the unnecessary UAV bombers losses.

Unmanned bombers take self-organizing cooperative reconnaissance path planning to avoid reaching hostile defenses of anti-aircraft fire region, which is labeled as "soft obstacles" on the cognitive map. Unmanned bombers show a character of obvious distributed system. Distributed unmanned vehicles takes cooperative reconnaissance attack military tasks in autonomous dynamic battlefield environment with high degree of unknown. Through autonomous cooperative path planning, an organic integration of no center control UAV reconnaissance and strike self-organization system is formed. Multiple UAV cooperative reconnaissance and strike missions require real-time path planning and navigation control strategy, which are mainly based on two independent tasks: a) "Cognitive map" is built as the reproduction of battlefield environment. The image information for "Cognitive map" is from UAV airborne image sensor scanning the battlefield environment. b) With the "Cognitive map" obtained, UAV reconnaissance and strike team will make path planning to complete the cooperative reconnaissance strike missions.

UAV arrives at region CELL $(\mathrm{x}, \mathrm{y})$ to search. The definition of threat probability $k(x, y, t) \in[0,1]$ stands for the probability that UAV in the promoter region of CELL ( $\mathrm{x}$, y) be destroyed. [2] If UAV searches CELL(x,y) at time $t$,

$$
\begin{array}{r}
k(x, y, t)=P(U A V \text { is destroyed }) \\
=1-\prod_{i=1}^{n}\left(1-p_{\text {kill }}^{j}(x, y)\right)
\end{array}
$$

In the above function $\mathrm{n}$ is the number of threats in CELL $(\mathrm{x}, \mathrm{y})$ in the above function[2]. As cognitive map stores threat probability, it is called the threat probability map, denoted as $K(t)$. It is initialized according to the type and location of the threats.[2] Because the threats are hypothesized static threats with the prior probability, the threat probability map is time invariant, denoted as $K(t)=K(0)$ [3]. Even at the start of the mission UAV does not know any threat, cognitive map can be gradually established with image sensors carried on UAV.

Each UAV owns three kinds of cognitive maps, such as target probability map $\mathrm{P}(\mathrm{T})$, threat probability map $\mathrm{K}(\mathrm{T})$ and uncertainty map $Z(t)$. Cognitive map is the summary of past information about the environment from each UAV. The initial values of the cognitive map, such as $\mathrm{P}(0), \mathrm{K}(0)$ and $\mathrm{Z}$ (0) are used to reflex the prior probability[2]. All of the targets must be on the ground, and there is no target on the region of lake. The initialization of $P(x, y, 0)=0, Z(x, y, 0)$ 
$=0$ and $\mathrm{K}(\mathrm{x}, \mathrm{y}, 0)=0$ indicate that targets or threats do not exist in these cells. When the UAV searches battlefield environment, new information is collected with UAV image sensor to update three cognitive maps. Each UAV owns three cognitive maps, such as target probability map P (T), threat probability map $\mathrm{K}(\mathrm{T})$ and uncertainty map $\mathrm{Z}(\mathrm{t})$. Cognitive map is the summary of past information about the environment from each UAV. The initial values of the cognitive map, such as $P(0), K(0)$ and $Z(0)$ are used to reflex the prior probability[2]. All of the targets must be on the ground, and there is no target on the region of lake. The initialization of $\mathrm{P}(\mathrm{x}, \mathrm{y}, 0)=0, \mathrm{Z}(\mathrm{x}, \mathrm{y}, 0)=0$ and $\mathrm{K}(\mathrm{x}, \mathrm{y}, 0)=0$ indicate that targets or threats do not exist in these cells. When the UAV searches battlefield environment, new information is collected with UAV image sensor to update three cognitive maps in real time. According to the distributed multiple UAV cooperative search model, each UAV i carries its own cognitive maps, written as $P^{i}(t), K^{i}(t), Z^{i}(t)$. The wireless communication between different UAV may lose some information or perform unexpected communication delay, therefor each UAV has different cognitive maps[2]. However, this paper assumes that each UAV can receive information from all other UAV without loss of information or communication delay. In short, wireless communication information of all different UAV received is the same in this study. Three kinds of cognitive map P (T), K (T), Z (T) could be applied to each UAV.

Each UAV should evaluate every possible path planning with cost equations. UAV selects the most optimal flight paths to take the mission efficiently. Therefore, the path planning decision problem in distributed multiple UAV team, is transformed into the optimization control problem.

Multiple UAV team cooperatively search the environment, while each UAV needs to know the state of the environment to make right path planning decision[2]. For each UAV, state of the environment is the UAV knowledge about the environment, which is mainly stored in the cognitive map of the UAV. Every UAV in the team flies in the same state of environment, written as

$$
x(t)=\{P(t), K(t), Z(t), v(t), u(t)\}
$$

UAV i makes path planning decision at time $t$ to choose the navigation path at time $\mathrm{t}+1$, written as $u_{i}(t+1) \in\{l, f, r\}$.[2] Especially $l, r, f$ represent different actions of UAV, such as turning right, turning left and heading straightly. The definition of vector $u(t+1)=\left[u_{1}(t+1), u_{2}(t+2), \cdots, u_{N}(t+1)\right]$ expresses the all the path planning decisions made by UAV at time $\mathrm{t}+1$. $u(t+1)$ is a function of time $\mathrm{t}$ about the state of the environment, written as

$$
u(t+1)=h(x(t))
$$

Function $h(\cdot)$ represents UAV path planning decision function, and it is decided by the path planning strategy of each UAV. Path planning decision $u(t)$ has been made by UAV at time $t+1$. UAV scans the new region CELL to detect targets and threats. UAV image sensor data is expressed by vector $b(t)=\left[b_{1}(t), b_{2}(t), \cdots, b_{N}(t)\right]$. At the same time, the threats in the region CELL will work to destroy the UAV in the attack range.

The action of threats destroy the UAV is written as vector $w(t)=\left[w_{1}(t), w_{2}(t), \cdots, w_{M}(t)\right], b(t), w(t)$ and the changes of navigation will decide the state of the environment $x(t+1),[4]$ written as

$$
x(t+1)=f_{s}(x(t), u(t), b(t), w(t))
$$

Equation (2) and Equation (3) define the dynamic characteristics of the system. Equations $h(\cdot)$ and $f_{s}(\cdot)$ are decided by the behavior strategies of UAV and threats. [5] The path planning decision of UAV will change the environment condition, and in turn, change state of the environment will change the relevant strategies of UAV and threats. UAV, threats and environment compose the whole system, whose dynamic characteristic is random. Because the characteristics of $b(t)$ and $w(t)$ are random.

To perform UAV cooperative reconnaissance mission, UAV should recognize the maximum number of targets within time $T_{f}$, while minimizing the threat caused by the loss of UAV. UAV reconnaissance system can collaborate path planning of UAV, mainly by maximizing the equation following:

$$
E\left\{G\left(x\left(T_{f}\right)\right)-\sum_{t=0}^{T_{f}-1} J(x(t), u(t))\right\}
$$

in which

$$
\begin{gathered}
G\left(x\left(T_{f}\right)\right):=\sum_{i=1}^{N} \pi_{\nu} \delta_{i}\left(T_{f}\right)+\sum_{(x, y) \in E} \pi_{t} \zeta\left(x, y, T_{f}\right) \\
J(x(t), u(t)):=\sum_{i=1}^{M} c\left(u_{i}(t)\right)
\end{gathered}
$$

$\pi_{v}$ represents the value of $\mathrm{UAV}$, while ${ }^{\pi_{t}}$ represents the value of target [6]. UAV here is of the same kind. Targets in the mission are with the same attribute. The first part of Equation (5) expresses the number of survived UAV when the task is completed after time $T_{f}$. The second part of Equation (5) expresses the number of targets detected during the task[3]. The relative size of $\pi_{v}$ and $\pi_{t}$ are important 
indicators that measure the survival of unmanned aerial vehicle (UAV) and task targets confirmed. $c(\cdot)$ in Equation (6) takes positive value. It represents the unmanned aerial vehicle (UAV) flies from one CELL to another, which is written as $u_{i}(t)$ [2]. Because this paper assumes that unmanned aerial vehicle (UAV) is of the same kind, and the flight to any direction costs the same time and energy. As a result, $c(\cdot)$ is constant. At each time step, different UAV members make path planning decision $u_{i}(t)$, in which $J(x(t), u(t))$ is constant . In these conditions, the UAV team searches the targets when maximizing the cost equation $G\left(x\left(T_{f}\right)\right)$ from Equation (5).

\section{The importance of cognitive map}

UAV team self-organized makes planning path, selecting an optimal path during the mission. UAV team has to know the information and state about battlefield environment, as well as information about distributed self-organizing path planning from other UAV members. In order to guarantee some UAV members are destroyed in the complex battlefield environment, the rest of the UAV members can still implement the task, each UAV have its own cognitive map, which stores the information about battlefield environment and mission objectives from UAV itself and the other UAV members from the team. The cognitive map is a comprehensive model. Cognitive map can guide UAV taking strike in battlefield environment, while updating the cognitive map constantly with the help of the image data collected from UAV airborne image sensor.

\section{Image sensor model of UAV}

A kind of simple and effective method for establishing cognitive map of the UAV is expressed in detail here. Through the increased image information from the UAV, cognitive map is constantly updated. The basic idea for establishing the map is reproduction of the UAV airborne image sensor data in the battlefield environment, locating the enemies important military targets as well as the nearby air defense military (typically antiaircraft gun and surface-to-air missile launcher), and then using evidence reasoning method to fuse the information during the search of UAV team.

The battlefield environment is divided into sub areas written as $\operatorname{CELL}(\mathrm{x}, \mathrm{y})$. There is a degree of uncertainty in each $\operatorname{CELL}(\mathrm{x}, \mathrm{y})$, written as $z(x, y, t) \in[0,1]$. It is on behalf of the CELL uncertainty. Each UAV i takes the cognitive map to store the uncertainty of knowledge about the battlefield environment. UAV updates its cognitive map with its own airborne image sensor data or that from other UAV team members (from wireless communication). This kind of cognitive map is used to measure the uncertainty for the battlefield, written as $Z^{i}(t)$ [2]. Uncertain degrees of each sub-region CELL in the map is expressed by numerical initialization between the mathematical interval $[\mathrm{C}, 1]$. It represents prior information from UAV in the sub-region CELL. CELL $z^{i}(x, y, 0)=1$ means UAV $\mathrm{i}$ is completely unknown about the sub-region CELL. UAV team has to search the CELL to find the positions and properties of the potential targets. Property expresses whether the new target is enemy important military facilities, or enemy air defenses and the performance of the weapon that UAV should avoid. CELL $z^{i}(x, y, 0)=C$ means it is not necessary to search this region. Because the hostile air defense artillery and missile launcher must be set on the ground, the unknown degree in the lake area in the battlefield environment is initialized to the $\mathrm{C}$.

Actually wireless communication between different UAV members may exist time delay and the loss of information. Therefore different UAV has different uncertain degree map. In order to make a simple analysis, wireless communication of UAV team members between each other is assumed reliable. Any UAV airborne image sensor data can be transmitted to other team members by perfect wireless communication without delay. So each UAV gets the information about subregion in battlefield environment, and then distributes it without delay to all members of the team. Different UAV members share the same uncertainty map, written as $Z(t)$.

When UAV team takes cooperative reconnaissance missions, airborne image sensor of each UAV scans the relevant state information in the sub-region of CELL. Because airborne image sensor may not be perfect, each image sensor can't provide $100 \%$ the relevant information of the UAV mission in the sub-region CELL. Therefore the unknown degree reduction ratio should be defined, written as $\eta_{i} \in(0,1]$. It expresses the part information ratio of the CELL by the image sensor of UAV i. The percentage of unknown reduction $\eta_{i}$ quantifies the credibility for image sensor data from UAV i reducing the uncertainty of CELL. The image sensor carried by each UAV team member is of the

same kind. $\eta_{i}$ describes all the uncertainty reduction ratios. According to the UAV airborne image sensor model defined above, the Dempster Fusion rule can improve the uncertainty map of UAV.

\section{Build a cognitive map with Dempster-Shafer Theory and evidential reasoning method}

The UAV can take the inaccurate and imperfect UAV airborne image sensor data to establish and gradually update the UAV environment model, as is called cognitive map. The service efficiency and quality of the cognitive map will affect the cooperative path planning behavior of UAV[1]. Environment model of UAV is established with the different sensors. The main method is to take the airborne image sensor observation of UAV in the sub-region CELL things as evidence, and fuse different sensor information by evidence reasoning technology. 


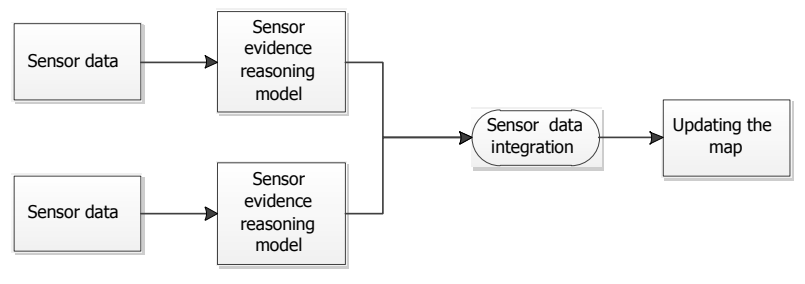

Fig. 1 The principle diagram of evidence reasoning for building cognitive map.

The common used methods to extract and share effective information are mainly several kinds of available evidence fusion methods. Fig. 1 illustrates the principle diagram of evidence reasoning for building cognitive map.

This paper takes the fusion method based on DempsterShafer theory to establish and gradually update the cognitive map. The cognitive map will guide UAV team members searching cooperatively and selecting the optimal path planning. This method is very suitable for sensor data that can be gradually acquired with the time.

The difference between Bias method and Dempster-Shafer method lies in the the reproduction modes for building cognitive map of UAV through the acquisition of airborne image sensor data[2]. With the help of Bayesian approach the cognitive map is probability built with airborne image sensor data, while with the help of Dempster-Shafer method the probability distribution of target in CELL is not considered. Different UAV sensor evidences can be distinguished, and UAV sensor evidences can be processes in a flexible way. Bias method doesn't know the prior measurements information.

Dempster-Shafer evidence method is considered to be the more general Bayesian approach. Compared with Bayesian methods, Dempster-Shafer evidence method has many advantages, especially it can distinguish whether a sub-region CELL has been searched by the UAV. Dempster-Shafer evidence method is taken to establish the cognitive map of the UAV. A detailed description of the process of UAV cognitive map is established. Through the simulation and analysis and discussion about the simulation results, it is demonstrated that in multiple UAV cooperative reconnaissance and strike missions, this method of establishing a target probability cognitive map based on Dempster-Shafer evidence method is feasible.

\section{Dempster - Shafer theory}

The core thought is unique and complete test set about problems, which is called the recognition framework, written as $\Theta$. The Credibility is expressed by real $[0,1]$. Basic probability assignment is expressed by $m: \Psi \rightarrow[0,1] . \Psi$ is subset of $\Theta$. The Power set of $\Theta$ is $\Theta=2^{\Theta}$. Function $m$ is the credibility of each element in $\Psi$.

$$
\sum_{A \subseteq \Psi} m(A)=1
$$

$$
m(\Phi)=0
$$

Basic probability assignment for element $\mathrm{A}$ is $m(A)$, which can describe the credibility of element $\mathrm{A}$. The total Unknown information is written as $m(\Theta)=1$. The evidence to improve the reliability of the element $\mathrm{A}$ is the whole probability distribution function of the subset, written as

$$
\operatorname{Bel}(A)=\sum_{\forall B: B \subseteq A} m(B)
$$

$\operatorname{Bel}(A)$ is the credibility for element A.[6] Not as the Probability theory, the sum of confidence range of element $\mathrm{A}$ and its complement $\bar{A}$ is not 1 . The plausibility of A means which evidence does not support $\bar{A}_{\text {[6] }}$, written as

$$
\operatorname{Pl}(A)=1-\operatorname{Bel}(\bar{A})=\sum_{B \cap A \neq \Phi} m(B)
$$

The Probability of A is between the interval of

$$
[\operatorname{Bel}(A), P l(A)]
$$

$\mathrm{Bel}$ and $\mathrm{Pl}$ are the Lower and higher reliabilities. The above interval is reduced to a point in the Bayesian equation. Two credible equations are determined by two independent sources (such as different UAV image sensors scanning to obtain data) .

\section{The cost equation of Unmanned aerial vehicle (UAV) cooperative reconnaissance choice of path planning}

In order to finish a higher efficiency reconnaissance mission, unmanned aerial vehicle (UAV) heads to the subregion CELL with the high probability of task goals through the Dempster- Shafer cognitive map. There is collaborative planning for path selection during the UAV reconnaissance.

\section{A. The target confirmed cost:}

This cost is mainly used for guiding unmanned aerial vehicle (UAV) heading to the subregion CELL with higher probability of task goal. Based on the Dempster - Shafer map building method, the corresponding [1] target confirmed the cost is:

$$
\rho_{f}(x, y, t+1)=e^{t(x, y, t)-i(x, y, t)}
$$

\section{B. The environment development cost [4]:}

This award is mainly used to guide unmanned aerial vehicle (UAV) heading to subregion CELL with higher unknown degree $i(x, y, t)$. With the building method of Dempster - Shafer map, cost $\rho_{e}$ expresses the ignorance of the unmanned aerial vehicle (UAV) for subregion CELL in the map storage:

$$
\rho_{e}(x, y, t+1)=i(x, y, t)
$$




\section{The avoiding threat cost and corporative cost [7]:}

With The definition of Bayesian map building method, long-term cost can be get as follows[2]: Dempster - Shafer map method is taken to establish a cognitive map, in which general long-term cost of subregion CELL $(\mathrm{x}, \mathrm{y})$ is determined by[6]

$$
\phi(x, y, t+1)=\max _{j \in\left[1,2, \cdots, 3^{T-1}\right]} \phi_{j}(x, y, t+1)
$$

The key problem to long-term cost is that allowing unmanned aerial vehicle (UAV) make the path decision a few more steps ahead, which can resist the existing information loss and delay information in wireless communication.

\section{Unmanned aerial vehicle (UAV) cooperative control method for avoiding multiple searching for a single CELL}

The unmanned aerial vehicle (UAV) cooperative search as a whole team to enhance the overall performance of the search task[1]. As a result, the main problem is how to prevent different unmanned aerial vehicle (UAV) searching the same CELL. Because each UAV optimizes its control behavior with its own information base. In the distributed collaborative control environment, other methods must be introduced for the synergy of unmanned aerial vehicle (UAV) control. One useful method is to take the other unmanned aerial vehicle (UAV) near the searching CELL as "soft obstacle", which applies a "force" for unmanned aerial vehicle (UAV) i to prevent unmanned aerial vehicle (UAV) i searching this CELL again. Unmanned aerial vehicle (UAV) i deletes this CELL as a "soft obstacle " from alternative paths.

With cognitive map, unmanned aerial vehicle (UAV) can avoid searching the subregion CELL that has been searched by other unmanned aerial vehicle (UAV). Choosing the smallest cost path planning with the simple evaluation of the cognitive map, unmanned aerial vehicle (UAV) can search, detect, and identify targets.

However, this method can't stop unmanned aerial vehicle (UAV) searching another CELL that other UAV will search. In this paper, the main idea of real-time cooperative search plan is how to prevent more than one UAV searching the same CELL. The already searched CELLs are identified as "soft obstacles". Other unmanned aerial vehicle is prevented choosing this CELL as path planning. At Each time step, UAV i confirms path planning decision under the influence of other unmanned aerial vehicle (UAV). UAV have to get rid of the CELL as "soft obstacle" because other unmanned aerial vehicle (UAV) have been searching the CELL. For example, unmanned aerial vehicle (UAV) i makes path planning to visit $\operatorname{CELL}(X, Y)$ at time $t$. The size of the force from UAV $j$ depends on Position and heading of UAV $j$ at time t. Through calculation for the shortest path that unmanned aerial vehicle (UAV) $\mathrm{j}$ heading to CELL (x, y), written as $l_{j}(x, y, t)$

The position of UAV j is $\lambda_{j}(t)$, and the heading is $o_{j}(t)$

. As UAV i and UAV $\mathrm{j}$ finish the search task cooperatively, if the path planning calculation predicts that UAV j searches CELL(x,y) with a higher cost, UAV i will visit this CELL. The force of UAV $j$ on UAV $i$ will be small. As a result, UAV i doesn't take this CELL as a "soft obstacle" to evade. UAV i searches the CELL actively. Conversely, if the path planning calculation predicts UAV $\mathrm{j}$ search CELL ( $\mathrm{x}$, y) with a lower cost, UAV $\mathrm{j}$ will visit this CELL (x, y). The "force" of unmanned aerial vehicle (UAV) $j$ on UAV i will be very large. UAV $\mathrm{i}$ will take this CELL as a "soft disorder" to evade. UAV $\mathrm{j}$ searches the CELL (x, y) with lower cost. Artificial potential field method defines "force" that UAV $j$ on UAV i to stop UAV i searching CELL ( $\mathrm{x}, \mathrm{y})$ as follows.

$$
F_{i j}(x, y, t)=\frac{1}{l_{j}(x, y, t)}
$$

The total "force" by other $\mathrm{N}-1 \mathrm{UAV}$ at time $\mathrm{t}$ when Unmanned aerial vehicle (UAV) i searches CELL $(x, y)$ is written as

$$
F_{i j}(x, y, t)=\sum_{j=1, j \neq i}^{N} F_{i j}(x, y, t)
$$

The "force" of UAV i searches CELL $(x, y)$ at time $t$ is depending on other N - 1 UAVs' position and heading. When the same CELL exists in different path planning options of UAV, different UAV members are affected by different "forces". Because position and heading is different between each UAV. At Each time step, unmanned aerial vehicle (UAV) takes evaluation equation to get the smallest value. UAV $i$ enters the CELL $(x, y)$ at time $t$ will be affected by other N - 1 UAV members' "force". Once the unmanned aerial vehicle (UAV) i enter this CELL that should be searched by other unmanned aerial vehicles (such as UAV j) with less cost, unmanned aerial vehicle (UAV) $\mathrm{i}$ will be punished.

\section{The simulation results}

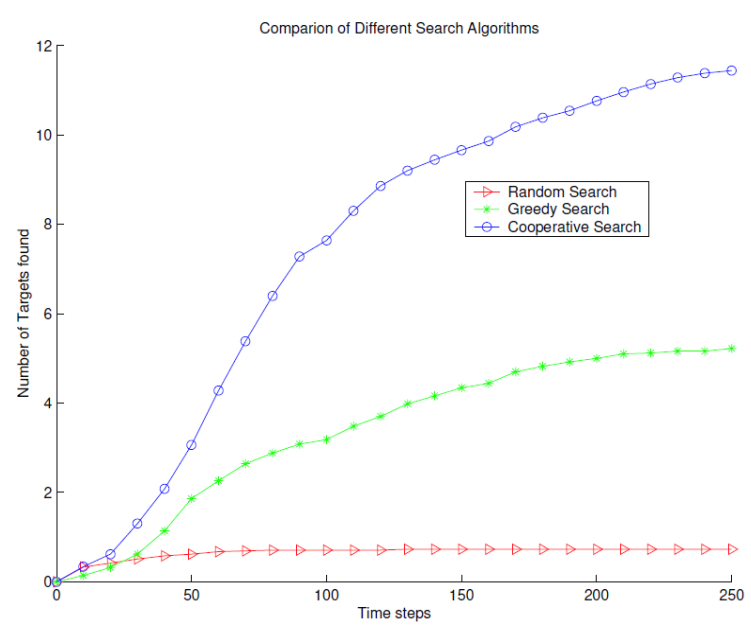

Fig. 2 The comparison among collaborative search, greedy search and random search. The number of potential targets founded as time increases. 
Simulation study shows the feasibility of using different improved evidential reasoning approaches. It shows that the reproduction of environment has an important influence on the performance of collaborative search. Representative examples are as follows: the team of $5 \mathrm{UAV}$ members searches the environment with scale of $20 * 20$. There are 20 objectives and 5 threats in the environment. There is neither prior information about terrain (lakes terrain with no targets or threats does not exist ), nor objective distribution information.

The UAV initial position and heading is invariable constant in all the simulation tests. The targets and threats have the same properties, and are randomly assigned to different subregion CELL. The attack area of threat is $\phi=2$. The probability of threats successfully destroying UAV is $p_{\text {kill }}=0.2$. UAV airborne image sensors take data truthful with the probability of $p_{c}=0.8$, and UAV airborne image sensor error warning rate is $p_{f}=0.1$. Simulation takes 250 discrete time steps.

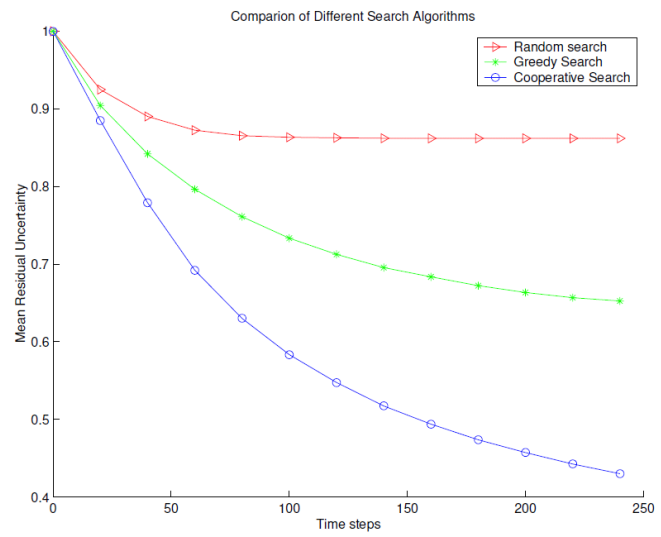

Fig. 3 The comparison of residual uncertainty relation with time among collaborative search, greedy search and random search.

Fig. 2 describes the different algorithms as a function of time for searching target. Fig. 3 shows that for different searching algorithms, the average residual uncertainty of the environment slowly decreases as the mission time increase. The average residual uncertainty of the environment is a good indicator for UAV covering search environment. Obviously, collaborative search algorithm improves the while search performance better than random algorithm and greedy algorithm.

The algorithm performance of UAV collaborative search is compared with random search and greedy search algorithms. The simulation results for random search algorithm shows that there is no available prior information about the distribution of the threats. UAV only changes the course of flight in the search area randomly. In the simulation of greedy search algorithm, the UAV will fly to subregion CELL with the smallest cost at each time step to confirm targets. At the same time UAV avoids to search the subregion CELL that have threats. With greedy search algorithm, UAV and the other team members share information. All the UAV members take the same cognitive map, though no cooperative path planning is done among the UAV members. Each UAV gets the smallest cost for its path planning, though the path choice may not be the best for the whole UAV team. As the proposed UAV collaborative path planning method, the UAV heads for the CELL with the smallest cost.

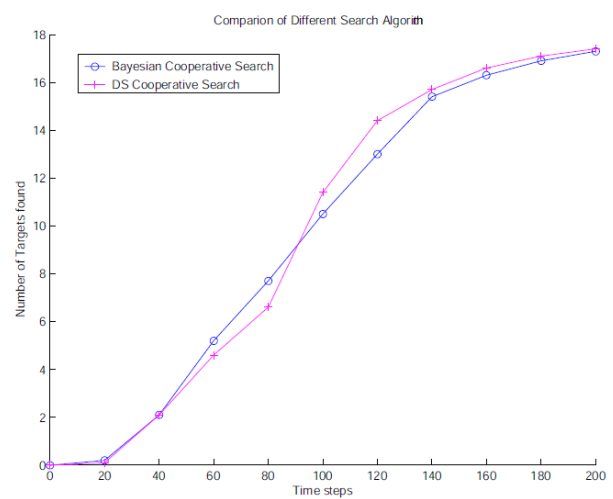

Fig. 4 When UAV carries higher accuracy of the airborne image sensor two different methods for building cognitive map give different performances.

A few simulation researches are set for comparing the different map design methods. Bayesian and Dempster Shafer map building method are established to make some simulation results.

The content of simulation experiment contains five UAV members, the scale of $20 * 20$ environment area, in which exist twenty mission objectives and two threats. There is no prior information of targets distribution. Targets and threats have the same properties with no individual differences. They are randomly distributed in the environment, while the initial position and heading of UAV is random given. The attacking area of threat is set to $\phi=1$ and the probability of destroying UAV successfully within the scope of threat is $p_{\text {kill }}=0.2$.

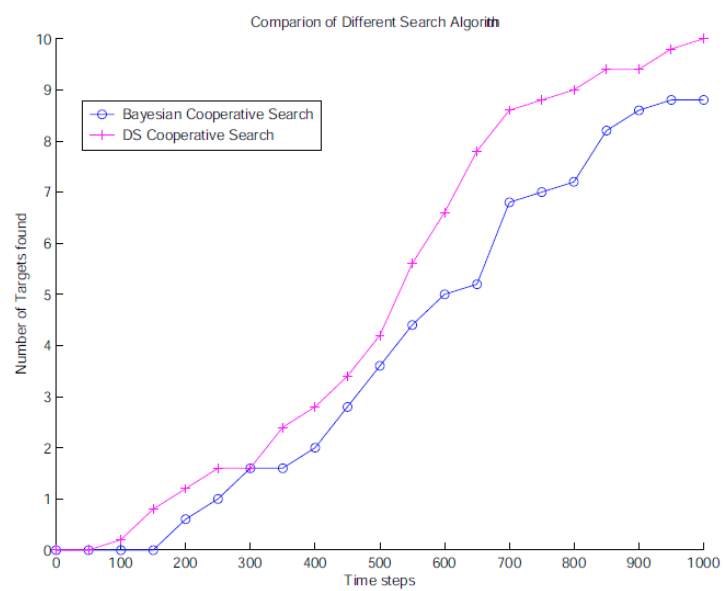

Fig. 5 When UAV carries lower accuracy of the airborne image sensor, two different methods for building cognitive map make different performances. 
The number of targets changes with the mission time. Fig. 4 describes when UAV carries higher accuracy of the airborne image sensor, two different methods for building cognitive map give different performances. On the other hand, Fig. 5 describes when UAV carries lower accuracy of the airborne image sensor, two different methods for building cognitive map make different performances.

UAV airborne image sensor makes no larger difference between the methods for building Bayesian map and Dempster - Shafer map. However, because the probability of conflict among the UAV airborne image sensors is very big, the lower UAV airborne image sensor accuracy makes Dempster Shafer map building method perform better than the other. It can help UAV find more mission targets. Because it considers the possibility that UAV airborne image sensor data may provide invalid information. It allows UAV to distinguish between two kinds of cases: a) Because the UAV team has not search the CELL and whether there is task target in the CELL is not known; b) Because the UAV airborne image sensor applies the contradictory information. UAV team can accomplish search task through the cooperative path planning strategy provided by this paper .

\section{Conclusion}

Unmanned aerial vehicle (UAV) builds cognitive map for the enemy important targets and anti-aircraft fire threats in the unknown environment. Then UAV tries to avoid the threats and head to important targets where the enemy locate. This paper studies how the unmanned aerial vehicle (UAV) in complex dynamic field environment make path planning to search the enemy's important targets. The standard for choosing the most appropriate path from alternative paths is based on cost equations. It is the smallest cost that the UAV can get. The study of self organization path planning is to analyze the four kinds of short-term costs and a long-term cost. Through adjusting the weight coefficient, all kinds of UAV path plannings in different battlefield environment can be achieved. The correct degree is considered in the process of establishing cognitive map with the UAV airborne image sensor data. Simulation results show that it is feasible to build two kind of cognitive maps separately with Bayesian theory and Dempster - Shafer theory. But when the error rate of the UAV airborne image sensor is higher, the Dempster - Shafer method for building cognitive map performs better.

\section{Acknowledgment}

This work was performed in the projects of both the National High Technology Research and Development Program of China (2011AA01A204) and the National Natural Science Foundation of China (91118002).

\section{References}

[1] Federal Aviation Administration Regulations http://www.faa.gov/regulations/Regulations.cfm

[2] Yang, Yanli. "Cooperative search by Uninhabited Air Vehicles in dynamic environment ", Proquest, 20111108

[3] Til Aach. "HDR-Microscopy of Cell Specimens: Imaging and Image Analysis", 2007 Conference Record of the Forty-First Asilomar Conference on Signals Systems and Computers, 11/2007

[4] M.M. Polycarpou. "Evidential map-building approaches for multi-UAV cooperative search", Proceedings of the 2005 American Control Conference 2005, 2005

[5] Yanli Yang, Ali A. Minai and Marios M. Polycarpou. "Decentralized Cooperative Search by Networked UAVs in an Uncertain Environment", Proceeding of the 2004 American Control Conference Boston, Massachusetts June 30 - July 2, 2004

[6] Zhang, L., J. Sheng, M. Xing, Q. Zhijun, T. Xiong, and Z. Bao "Wavenumber-domain Autofocusing for Highly Squinted UAV SAR Imagery", IEEE Sensors Journal, 2011.

[7] Bogdan Smolka, Cristian Canton-Ferrer, Marek Szczepanski, KonradWojciechowski, "EVALUATION OF THE EFFICIENCY OF ROBUST ANISOTROPIC DIFFUSION SCHEMES", Zakopane, 25-29 Sept. 2002 